ISSN 1112-9867

http://www.jfas.info

\title{
A MODEL FOR ACHIEVING URBAN RESILIENCY IMAGINARY AND EXPLAINING ITS RELATION WITH POVERTY
}

\author{
M. H. Sharifzadegan ${ }^{1}$, R. Ramezani ${ }^{2 *}$ \\ ${ }^{1}$ Associate Professor in urban and regional planning, Shahid Beheshti University, Tehran, Iran \\ ${ }^{2}$ Phd. Student in urban and regional planning, Shahid Beheshti University, Tehran, Iran
}

Received: 03 February 2016 / Accepted: 17 June 2016 / Published online: 01 September 2016

\begin{abstract}
In order to reduce the risks of disasters, we need to establish theories to make seemingly disordered events, as logical phenomena. In fact, theory is like a map for reducing, answering and recovering from disasters. This subject is by the way, an exception because theory needs to be verified based on its immediate usage. This is a kind of necessity based on which problems of disasters, immediate response and reduction of risks must be answered so we cannot just make the theory and wish for it to become practical some day in future. Resilience in Urban and Regional Studies discussions is in search of special local properties that decrease vulnerability of urban areas. This is rooted in disaster confrontation studies so notion of resilience has more main limitation in answering the social-economic challenges. One of the most important challenges is confronting with poverty notion. Poverty, on one hand, reduces resilience because of increasing vulnerability, and on the other hand, poor locals regenerate vulnerable environments due to their high social correlations.

The present study aims at investigating and analysis of theoretical and practical features in addition to recognized the dimensions of these two notions and their relation, plus extracting the main common concepts and indices of the relationship. Findings of the study show that governance concepts, social capital, social learning, social participation, institutionalism and vulnerability are among the intermediate rings in defining the relationship between poverty and resilience.
\end{abstract}

Keywords: notion, resilience, poverty, vulnerability, disasters

Author Correspondence, e-mail: author@gmail.com

doi: http://dx.doi.org/10.4314/jfas.v8i3.34 


\section{1-INTRODUCTION}

More than half of the world's population lived in urban areas at the end of twentieth century and beginning of the 21th. Urban areas are the running potential of national growth and play an important dynamic role in different governments. However, natural disasters during the history, have made problems for urban life and caused impairments in their systems' dynamism. Climate changes, earthquakes, and emergencies caused by human-made structures vulnerability, are among the most pressing problems on people, and threaten the boom of the cities. Tsunami of India in 2004, Katrina and Rita hurricanes in 2005 and global warming are all signs of vulnerability of urban areas against natural disasters. This becomes more important when we know that in recent years, damages of the disasters has been about600 billion $\$$ on more than 3 billion people of which 750000 died (Birkmann, 2006).

These trends show lack of resilience of human societies against natural disasters. A plan framework called HYOGO (which was introduced in HYOGO international conference in Cube, Japan (2005) on crisis control) was introduced during 2005 to 2015. After using HYOGO framework, the main purpose of risk planning has been to strengthen resilience instead of reduction of vulnerability (Mayunga, 2007). In fact, resilience is a way for strengthening the societies to use their capacities and returning to stable situation of before the disasters. In more developed countries, there are observed considerable changes in attitude toward dangers so that the prevailing view is changed from mere focus on vulnerability reduction to resilience increase against accidents and disasters. Disasters are mostly considered as natural acts. But this view is not true. Main factors that influence these risks are, human and social vulnerability that accompany with the total capacity of answering or reduction effects of natural disasters. Therefore, risk reduction programs must build and reinforce the features of resilient societies (Cutter et.al, 2008: 3).

Poverty is the single most important factor in determining disaster vulnerability that has been increased due to urbanization. It is a worldwide wish to reduce urban poverty. In fact, cities are considered to play the role of economic growth but sometimes they confront big challenges such as overcrowding and diseases.

There have been considerable attempts to confront these problems by international organizations and local governments. But it is possible to reduce this issue via both considering societies' resilience against natural disasters and poverty. Before any planning and programming for reduction of these phenomena, we should identify the relationship between these two notions. It seems that in many studies, there is accepted to be a significant 
relationship between disaster resilience and urban poverty as a default. But this needs to be investigated carefully to find actual and practical strategies to confront them.

\section{2-PURPOSE AND METHOD}

As mentioned earlier about epistemology concepts of poverty and also the importance of finding the relationship between them, the aim of our study is to find the relationship between resilience and poverty in cities and urban planning framework. General questions of the study are divided into two categories of "what" and "why":

What questions:

- What is the notion of resilient in city and neighborhood scope?

- What are the effective indexes on local and urban resilience?

- What is the notion of poverty in city and in local?

- What is the relationship between poverty and resilience in local scope?

Why question:

Why urban poverty is effective on locals' resilience?

In this way, method of the study is descriptive based on theoretical and practical texts of world literature.

\section{3-background of the study}

A study by Cutter et al. was performed at 2010 titled "Indicators of Resilience events for case study of baseline conditions" in which Alabama, Florida, Georgia, Kentucky, Mississippi, north and South Carolina, and Tennessee were included. It was about the spatial distribution of disaster resilience in 736 provinces at FEMA region.

In another study by Cutter et.al, they determined the resilience scale of three regions of Metropolitan Galfport-Biloxy, Charleston and Memphis. The important issue of these two studies was that both of them showed the usage of descriptive method based on experiments in order to determining the regions with least resilience in different geographical levels.

In another study, resilience display project of the society of coastal Newberys was done at 2010. This project used two innovative tools including CCVAP protocol and resilient questionnaire both of which were provided by National Oceanic and Atmospheric Administration.

This protocol is a GIS-based evaluation method that helps local governments to investigate vulnerability of their societies against river floods now and in future.

Verrucci et al. (2012) studied indicators of urban resilience investigation against earthquake. They completed the used indicators of Cutter et al (2008) and Broneo et al. (2003) and 
provide a theoretical framework for determination of topical regions and then presenting the suggested indicators.

Normandin (2012) tried to find the indicators used for studying vulnerability and resilience in urban areas. Also in the study of Theo (2013) entitled "an integrated framework for investigation of society resilience in disaster management" they studied the variables such as environment, society, government, infrastructures and economy.

But another study was performed at 2009 in order to find a technique for analysis of poverty in small neighborhoods including Carlos Delasprila in Costa Rica compared to medium sized city of Liberia. This technique is evaluated regarding the capacity of it for determination of the relationship between social home-making, poverty and inequality, effects of administering chosen policies and differences of political goals. This technique helped to find the effects of housing policies in poverty reduction in Liberia.

In another study by Isa Baud, Sridharan and Karin Pfeiffer(2007) entitled "Illustration urban poverty to the local government in a metropolis in India (Delhi)" they used "Livelihood capital framework" to illustrate the urban poverty and make a new indicator for multiple deprivation and determine the targeting a region or a part by decision makers.

In another study by Woldemariamand Limia (2003) in eleven selected African countries, they studied indicators of drawing poverty maps of Africa.

Thousand and Mack and Lansley tried to use a subjective method for measuring the relational poverty. Thousand asked participants to express their ideas about enough income for satisfying their needs and expenses. Their answers showed that their required income is 61 percent higher than what government had identified (Giddens, 2007, 451).

Also, Mack and Lansley performed a survey based on findings of Thousand, in order to find out the acceptable required items for ordinary people life that 26 one of the items were selected. If families lacked at least three of these 26 items, they were considered poor and if they lacked 7 of them, they were considered to be seriously poor (Mack \& Lansley, 1985, 1992).

In 2007, Australia government accomplished a study with Melbourne University in which financial problems of Australians during 2001 and 2002 were studied under leadership of Marquez. Findings of the study were divided into different dimensions including occurrence and persistence, gender, age, nationality, family type, financial situation and children, education, job market experiences, income, wealth and debt. The main investigation of this study was to find the relationships and influences of poverty and disaster risks. UNHABITAT performed a study on finding the relationship between these notions (2012) and 
focused on environmental changes influencing vulnerability and different dimensions of urban poverty (UNDP \& UN-HABITAT, 2012).

In another report, by United Nations at 2008 entitled "relationship between environmental risk reduction and poverty reduction", they studied the relationship between risks' influence as an independent variable and poverty amount in an environment. It is worth to mention that this report studied countries such as Bangladesh, Bolivia, El Salvador, Honduras, India, Indonesia, Kenya, Libya, Mozambique, Nepal, Pakistan and Peru (ISDR \& UNDP, 2008).

A study called "the relationship between natural disasters and poverty: case study Fiji” (2009) by Padmalal et al, for United Nations organization, developed a method for experimental investigation of relationship between poverty and disasters in Fiji. A combination of quantity and quality methods are used in this study:

- Quantitative and qualitative analysis of nature, and disasters frequency

- Quantitative analysis of economic structures, economic development trends based on Fuji statistics, and statistics of reports of international institutions such as World Bank and Asian development bank.

- Analysis of trends and qualitative analysis of family commonwealth, based on statistics ofFuji government, international bureau of poverty and development, economic development bank reports and world bank in UNDP academic report.

- Quantitative Economic investigation of the mutual relationship between poverty and disaster, focusing on national integrated analysis

In a study by Andavarapu and Arefi (2015) they investigated the slum settlements in recent years of Mumbai, India and Rio, Brazil which challenges people in poor neighborhoods that they are passive and victims of the disasters and focus on their abilities to adapt their environments and finally concludes that the social capital in these slums lead to increase in resilience of these communities.

\section{Theoretical framework}

\section{Resilience notion}

Resilience is a combination of different disciplines including ecosystem stability (Holling, 1973; Gunderson, 2009), engineering infrastructure (Tierney and Bruneau,2007), economics (Rose, 2004), psychology (Lee et al, 2009), behavioral science (Norris, 2011) and reduction of the disasters risks (Cutter et al, 2008). As a result, we can claim that resilience is a common agenda among people who are worried about financial threats, political and disasters threats, and conflict and climate threats for development. 
The aim of the resilience planning is to make sure that shocks and tensions (individual or combined) do not result in long term reduction of development process that is measured by human development indicator, economic growth and other measurement methods (Mitchell \& Harris, 2012).

Ability of groups or communities for adaptation with external tensions and chaos, ability of social units to reduce risks, restoration activities to reduce social disruption, utilization of opportunities via communication improvement, knowledge about risks and preparation, development and administering damage management, insurance and transfer of information to help to restoration process. Capacity reversible can be improved using physical and economic resources, ability of people and societies in confronting danger conditions, enabling internal power for adaptation and flexibility (Adger, 1999).

The high degree of connection between social and ecological systems is certain, but theorists with different backgrounds, have different ways of understanding resiliency that lead to different ideas about the components, characteristics and indicators systems. Theories are arisen that have seen and the resilience of social and ecological formed. As well as those they are intended to be connected.

\section{Notion of poverty}

At the present time, more than half of the world's population live in cities that is increasing in developing countries (World Bank, 2013). The findings of global monitoring report, published by IMF, estimates that by 2030, 1.4 billion people will be living in developing countries of which $96 \%$ will be in urban areas (World Bank \& IMF, 2014). More than $32 \%$ of urban population of developing countries live in poor neighborhoods. This refers to urbanization huge scale and resident shortage. In some cities such as Mumbai, India, there are $41.3 \%$ of poor people (Andavarapu \& Arefi, 2015).

There's a complex relationship between urbanization and poverty. On one side, urban poor people confront more sanitary problems compared to rural residents and pay more money for their essential water and wastewater services. On the other side, urbanization provides higherpaid job opportunities, school access, and better sanitary services. With these complexes, urbanization rate and poverty situation is changing toward cities. It is estimated that poor population in cities worldwide is increasing 10\% (Un-habitat, 2010, p.30).

It is not easy to provide a definition of poverty because it is a multi-dimensional phenomena. Some social indices such as life expectancy, infant mortality, and nutrition, food budget of the family, illiteracy, school enrolment, sanitary clinics and drinking water access are among the important factors in defining poverty (Wratten, 1995). This definition was developed by 
Amartiasen who won Nobel Prize in 1998 and considers poverty as deprivation of capabilities. He believes that "poverty is identified by capability deprivation (this strategy focuses on deprivations which are in spite of income, important from institutional point of view)". This approach doesn't reject low income as an important poverty factor, but it emphasizes that high income doesn't guarantee that the person is not deprived of anything.

Attitude feature is an alternative for individual behavior conceptualization, evaluation of commonwealth and determination of political purposes that is formed based on rejection of utilitarianism as a measure for commonwealth and maximizing the benefit as a behavioral supposition which is rooted in utilitarianism ethical base criticism. It is claimed that the only defense base of utilitarianism is to consider it based on the meaning of benefit to perform an interpreted purpose.

Undoubtedly, definition of poverty as an inclusive concept for all conditions and all times is impossible since it is a relative concept that's definable based on its spatial and temporal conditions. Nevertheless, human poverty is defined as the lack of ability to overcome illiteracy, hunger, reduction of age and life expectancy, lack of hygiene, lack of possibility to prevent from curable diseases, of which indirect measures are:

Lack of access to goods, services, infrastructures, fuel, hygiene, education, communications and drinking water, all of which are necessary for increasing abilities of humankind (Mehta \& Shah, 2001).

We should add civic participation and individual freedoms that are among cultural, social and political rights of people (Jitsuchon, 2001, p.9).

\section{5-Findings of the research: definition of the relationship between disaster and poverty notions}

There are lots of evidences about the relationship between disaster risk and poverty. Findings of interviews with poor people worldwide (World Bank, 2000), UNDP report at 2004 about relationship between disasters and development, and Britain international development department report about the relation between development and disaster (in long term) all show the relationship between disasters and poverty (Lal, Singh \& Holland, 2009). Poverty and disaster vulnerability are interrelated. Poverty adds to sensitivity of people against disasters and also increase the effects of disasters on their comonwealth. Disasters reduce comonwealth of people and add to their poverty. Anyway, the outcome of a mutual relationship between poverty and disasters, is among many factors and the experimental relation between poverty and disasters depend on a complex network of economic, environmental, social and political forces. The mutual strong relation between human developmen condtions and disasters, 
shows a multi-dimensional attitude focused on reduction of poverty and sensitiviety of society against dangers and risks in order to increase its resilience. (Baker, 2012).

Now, after investigating the history and experiences of the study, it seems that some notions are more influencial in explanation and definition of the relationship between resilience and poverty that will be studied in details.

\section{Definition of the relationship between governance, poverty and resilience}

Investigation of cities' experiences in decreasing disasters risk in good governance frame work can be a good starting point for better recognition of the relationship between urban governance and climate resilience and potential for governing well a city in order to achieve a stable climate resilience. There should be performed deeper analysis for understanding the amount of connection between urban governance and resilient climate development and finally, decreasing the risk of disasters for vulnerable population of the cities. There must be enough and suitable infrastructures, services and building regulations for making a strong framework for climatic resilient (CORPS, 2008). The following reasons are important for existence of a stable resilience:

- Multiple-outcome confusions, such as accidents with death and economic losses.

- In order for decision makers to understand problems in a recognition framwork with complete and valid information plus proper analysis about them.

- Accessing to the legal tools, innovational solutions and resources able to solve the problems

- Because public participation can solve the problems

- Because problems can be solved among many participants who are beneficiary.

Resilience can be improved via Bottom-up approach or top-down approach. Society-centered programs and successful innovations include rapid alarm systems and saving schemes, however, building a resilient society needs public actions too. Frequency of climatic disasters that increase poverty and urban poor vulnerability, will lead to increasing information and knowledge in governments for the need of attention and stable adaptation with climatic changes risks. In order to adapt with these societies, they need to be supported by public and private organizations. Cities should firstly provide conditions and opportunities for the poor people and vulnerable communities (UNDP \& UN-HABITAT, 2012).

\section{Definition of the relationship between institutionalism, poverty and urban resilience}

Every village or city may experience or undergo changes or transformations that may influence their different structures. Some cities and villages can be adapted with the changes 
but some take more time to get adapted. Resilience notion presented some ideas for easier understanding these differences.

Institutions are made of legal and formal regulations (constitution, statute book, customary law and regulations), informal constraints (customs and behavioral norms and self-imposed behavioral regulations) and their enforcement guarantee. Institutions reflect actors beliefs- or at least actors that are able to form the beliefs- so they are beyond the beliefs, language and cultural heritage (North, 1991).

Formal laws are highly directed which contain constitutions; regulations and everything owning these properties that can be defined precisely. Informal norms of our behavior make more problems for us because they are not precisely defined. They are the ways things are performed and so, they are very important. We have different types of formal regulations but a small part of them form out daily behaviors and activities. For different reasons, norms are more essential than formal regulations therefore, at first level, we have formal regulations and informal norms and at next level, we have their executive features (North, 2003).

Talking about disasters, an institution that propagates resilience, must be in relation with government, civil and traditional institutions plus observing their own behavioral norms and traditions to improve systems of disaster risk reduction (DDR). An institution is considered as a tool for action that is beyond its own abilities and values.

\section{Relationship between social learning, poverty and resilience}

People-centered development and humanitarian programs have in their own social learning and institutional inherent. The resilience of a community is inextricably linked to the condition of the environment and the treatment of its resources; therefore the concept of sustainability is central to studies of resilience. Within the context of natural disasters, sustainability is defined as the ability to tolerate and overcome damage,diminished productivity, and reduced quality of life from an extreme event without significant outside assistance. An environment stressed by unsustainable practices may experience more severe environmental hazards. There has been a call for a shift from ad hoc, disaster-driven, and reactive systems and policies to a proactive, threat-driven, and mitigated focus (Edger et al 2005). These efforts not only make sense for reducing the impacts of environmental hazards, but they are also much more in line with the generational equity concerns inherent in sustainability science. The most recent international efforts are those embodied in the Hyogo World Conference on Disaster Reduction held in 2005in Kobe, Japan. The Hyogo Framework for Action identified both the need for and ways to build resilient communities by integrating disaster prevention, mitigation, preparedness, and vulnerability reduction perspectives into 
sustainable development policies; (2) increasing local capacity (institutions and mechanisms) for building hazard resilience; and (3) incorporating risk reduction into the design and implementation of emergency preparedness, response, recovery, and reconstruction programs in affected communities (Cutter et al., 2008b).

\section{Definition of the relationship between society companionship, poverty and resilience}

Resilience refers to the capacity of a dynamic system to adapt successfully to disturbances that threaten system function, viability, or development. Based on the international experiences, one of the methods of increasing ability of urban systems for resistance and rapid restoration in dangerous events, access to urban systems from enabled and motivated citizens for participation in different stages of crisis management cycles, prevention and decreasing risks, preparation, confrontation and restoration that are mentioned in HYOGO 2005-2015 framework to provide resilience for nations and societies who are members of United Nations organization. This is an integrated framework that considers the roles of governments, regional and international organizations and calls civil society, volunteer organization, and private sector for joining these attempts.

One of the priorities of HYOGO administrative framework that focuses on this issue, is to provide institutional capacities at local and national levels for reduction of risks, identification and evaluation and supervision of risks of disasters, increasing understanding and knowledge, innovation and education to build security culture and resilience at all levels, decreasing risks and increasing preparation for rapid response to disasters. (UNISDR, 2005).

Based on the definition of World Bank, resilience is supported or thwarted by direct effects of risks and resources, as well as by moderating processes of protection, vulnerability, and differential susceptibility. Resilience is dynamic; it emerges from many interactions within and between systems in a given cultural, developmental, and historical context that collectively influence the capacity of an individual system to adapt successfully to challenge. Although resilience research often has focused on the behavior of individuals, contemporary models of resilience encompass multiple levels of function and acknowledge the interdependence of interacting systems, ranging from molecular to societal levels of analysis across individuals, families, peer groups, schools, communities, governments, and cultures. Cultural influences on resilience are gaining traction amid growing recognition that interventions should be tailored to the unique strengths, vulnerabilities, and values of specific contexts, and also that different cultures may have traditions and practices that can inform resilience theory. Resilience remains an inspiring and informative framework for implementing positive psychology in practice. 


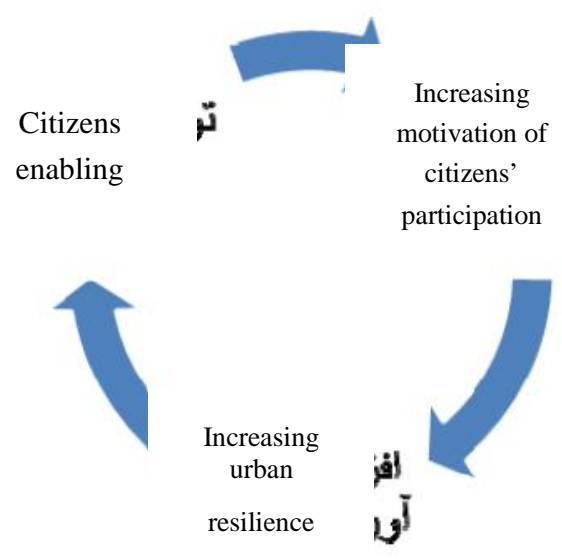

Fig.1. relationship between empowerment, citizen participation and increasing the urban resilience

\section{Relationship between social capital, poverty and resilience}

National or international NGOs, local government or CBOs have mobilized people in slums in order to confront with natural (fire/flood) and human-caused (discharge/crime) disasters and risks.As a result, linking capital is necessary for resilience and stability of these areas (Andavarapu \& Arefi, 2015).

Researchers such as Walsh $(2002,2006)$ define elements and special processes for family resilience and arranged bases for it. Walsh defines three areas including:

1-belief systems

2-family organization templates

3-relational processes.

On the other hand, these areas are defined based on the special skills related to resilience. Juby \& Rycraft (2004) focused on supportive factors that enable families to confront poverty stresses. They identified four individual, familial and social factors that help families' resilience:

1-an internal control center believing that individuals or families are enabled to influence their environment.

2-spiritualitythat develops individuality and life purpose

3-social comparison

4-social support including emotional support, information support, social companionship, tool support. 
According to the study of Arce \& Mullin (2008) on poor families' resilience, they concluded that poor families will be more resilient when:

-They search for support or are supported as a method for building internal relationships

- They believe in themselves, their society and spiritual world of which are fed with.

- They take operating steps for having self-control on their life.

These are inseparable processes with internal relations that strengthen each other.

\section{5-CONCLUSION}

Urban resilience was introduced in spatial planning in search of finding a solution for controlling disaster stresses in societies and then resilient cities, at 1990s. Nowadays, resilience studies are focusing on governance adaptation ability and not only consider vertical relations in planning systems, but they take into consideration horizontal networks to form cities for reduction of serious changes.

It is essential to consider urban resilient for two reasons:

1-changes are adapted without disastrous failure

2-it enables people to live in places that are not in stressful areas.

Theoretical negotiations of resilience are developed in four decades. But urban resilience studies on spatial planning started in recent years. Nowadays, planning is still seeking for strategies in order to strengthen social and physical networks to make them flexible.

In poor areas of developing countries, life and work in risky environment plus content factors such as poor governance and inadequate infrastructures are influential in their vulnerability against unfavorable climatic conditions. For example, in Lagus, Nigeria, poor people choose residential areas prone to flooding for their cheap prices and familial links and job opportunities (Leichenko \& Silva, 2014).

Understanding urban resilience as a new institutionalization provides promising prospects for social studies on urban changes. "Urban" conceptualization as a hybrid or complex multidimensional system -system thought is a special property of resilience studies- directs us to seek for different dynamics of interfering and hybrid processes in vulnerability, crisis and change. This systemic approach is suitable for government studies full of normative expectations of how the government should be. Theoretical knowledge and resilience concept can help understanding changes in spatial relationship of complex systems and fill the gap in institutional theory. But on the other hand, with development of analytical stages, new institutionalized situation can help determining the properties that resilient the urban system. As a result, understanding the development as a combination of adaptive embedded cycles 
can help understanding changes and stability. In contrast withurban governance systems, adaptive cycles can be seen as autonomous systems. Part of conceptualization of adaptive cycles is that they can only become adapted with exceptional situations of multiple challenges.

Attempts for increasing social knowledge and conceptualization of urban and regional resilience needs to be continued especially when we talk about power, institutional limitation and national regulations. In any ways, combination of resilience and systemic thinking with new institutionalism ideas can be promising. We don't know yet that how these changes are managed but in regional and urban development we need to pay more attention to the relationship between urban development and social and spatial justice.

\section{6-REFERENCES}

- Adger, W. N. (1999). Social vulnerability to climate change and extremes in coastal Vietnam. World Development, 27(2), 249-269 .

- Adger, W. N. (2006). Vulnerability. Global Environmental Change, 16(3), 268-281 .

- Andavarapu, D., \& Arefi, M. (2015). Resilient Slums: Role of Social Capital. Tekton, 2(1), 38-53 .

- Baker, J. L. (2012). Climate change, disaster risk, and the urban poor: cities building resilience for a changing world: World Bank Publications.

- Birkmann, J. (2006). Measuring vulnerability to natural hazards: towards disaster resilient societies.

- Burton, C. G. (2012). THE DEVELOPMENTOF METRICS FORCOMMUNITY RESILIENCE TO NATURAL DISASTERS. University of South Carolina .

- CORPS, M. (2008). Climate Resilient Development case study - A governance approach to building urban climate resilience. Retrieved from

- Cutter, S. L., Barnes, L., Berry, M., Burton, C., Evans, E., Tate, E., \& Webb, J. (2008a). COMMUNITY AND REGIONAL RESILIENCE: PERSPECTIVES FROM

- HAZARDS, DISASTERS, AND EMERGENCY MANAGEMENT Retrieved from

- Cutter, S. L., Barnes, L., Berry, M., Burton, C., Evans, E., Tate, E., \& Webb, J. (2008b). A place-based model for understanding community resilience to natural disasters. Global Environmental Change, 18(4), 598-606 .

- Devas, N. (2014). Urban governance voice and poverty in the developing world: Routledge. 
- Huq, S., Kovats, S., Reid, H., \& Satterthwaite, D. (2007). Editorial: Reducing risks to cities from disasters and climate change. Environment and Urbanization, 19(1), 3-15 .

- ISDR, \& UNDP. (2008 .(Linking disaster risk reduction and poverty reduction : good practices and lessons learned : a publication of the Global Network of NGOs for Disaster Risk Reduction. Retrieved from

- Jitsuchon, S. (2001). What is Poverty, and How to Measure it? TDRI quarterly review, 15(3), 6-10 .

- Lal, P. N., Singh, R., \& Holland, P. (2009). Relationship between natural disasters and poverty: a Fiji case study: SOPAC.

- Lang, T. (2011). Urban Resilience and New Institutional Theory-A Happy Couple for Urban and Regional Studies? German annual of spatial research and policy 2010 (pp. 1524): Springer.

- Leichenko, R., \& Silva, J. A. (2014). Climate change and poverty: vulnerability, impacts, and alleviation strategies. Wiley Interdisciplinary Reviews: Climate Change, 5(4), 539.556

- Manyena, B. (2009). Disaster resilience in development and humanitarian interventions. Northumbria University .

- Mayunga, J. S. (2007). Understanding and applying the concept of community disaster resilience: a capital-based approach. Summer academy for social vulnerability and resilience building, 1,16 .

- Mehta, A. K., \& Shah, A. (2001). Chronic poverty in India: overview study. Chronic Poverty Research Centre Working Paper(7 .)

- Mitchell, T., \& Harris, K. (2012). Resilience: A risk management approach. ODI Background Note. Overseas Development Institute: London .

- Mullin, W. J., \& Arce, M. (2008). Resilience of families living in poverty. Journal of Family Social Work, 11(4), 424-440 .

- North, D. (1991). Institutions. Journal of Economic perspectives, 5(1), 640-655 .

- O'Keefe, P., Wisner, B. (2005). African Drought: The State of the Game. In: Richards, P. (Ed.), African Environment: Problems and Perspectives. Retrieved from London :

- Taşan-Kok, T., Stead, D., \& Lu, P. (2013). Conceptual overview of resilience: History and context Resilience Thinking in Urban Planning (pp. 39-51): Springer.

- Un-habitat. (2010). State of the world's cities 2010/2011: bridging the urban divide: Earthscan. 
- UNDP, \& UN-HABITAT. (2012). The Asia-Pacific Issue Brief Series onUrbanization and Climate Change. Retrieved from

- UNISDR. (2005). Hyogo F ramework for A ction 2005-2015: Building the resilience of nations and communities to disasters. Retrieved from

- White, P., Pelling, M., Sen, K., Seddon, D., Russell, S., \& Few, R .(2005) .Disaster risk reduction: a development concern. London: DfID .

- WorldBank, \& IMF. (2014). Ending Poverty and Sharing Prosperity. Retrieved from

- Wratten, E. (1995). Conceptualizing urban poverty. Environment and Urbanization, 7(1), 11-38 .

- Yodmani ,S. (2001). Disaster risk management and vulnerability reduction: Protecting the poor: The Center.

- Young, O. R. (1994). International governance: Protecting the environment in a stateless society: Cornell University Press.

\section{How to cite this article:}

Sharifzadegan H M, Ramezani R. A model for achieving urban resiliency imaginary and explaining its relation with poverty. J. Fundam. Appl. Sci., 2016, 8(3), 1258-1272. 